\title{
Simulation Model for OBS Contention Avoidance Routing Strategies
}

\author{
Alvaro L. Barradas and Maria do Carmo R. Medeiros \\ Center of Electronics Optoelectronics and Telecommunications (CEOT), \\ Universidade do Algarve, Campus de Gambelas, 8005-139 Faro, Portugal \\ \{abarra, cmedeiro\} @ualg.pt
}

\begin{abstract}
Optical burst switching (OBS) provides a feasible paradigm for the next IP over optical network backbones. However, due to its bufferless nature, OBS efficiency can be reduced by resource contention leading to burst loss. Several methods have been proposed to address this problem, most of them relying on reactive mechanisms which increase the complexity of core nodes, hampering scalability. In this work we consider a preventive traffic engineering approach for contention resolution which provides source routing with the objective of minimizing contention at the transmission links considering only topological information. This paper presents a simulation model aimed at the evaluation of different offline routing strategies in terms of burst contention. The simulation model is used to compare the performance of different novel path selection strategies with the traditional shortest path routing approach. Results confirm that the proposed strategies are effective in reducing the overall blocking and the model is feasible for the proposed QoS evaluation.
\end{abstract}

Keywords: Optical networks, Network architecture and design, Optical burst switching, Contention avoidance, Routing strategies, Simulation.

\section{Introduction}

Optical burst switching (OBS) [1], [2] has emerged as an efficient switching paradigm for the core of IP over optical networks. OBS avoids the inefficient resource utilization of Optical Circuit Switching (OCS) and the requirements of buffers, optical logic processing and synchronization problems of Optical Packet Switching (OPS). In OBS the basic transport unit is the burst, an aggregate message that can be regarded as an optical "super packet" containing multiple IP packets going to the same egress node and (if used) grouped by some Quality of Service (QoS) criteria. Bursts are assembled at the ingress nodes and their transmission is preceded by dedicated setup messages, transmitted on a dedicated control channel with the purpose of reserving bandwidth along the path for the upcoming data bursts. Based on the information carried by the setup messages, the intermediate nodes reserve switching resources along a preconfigured path, providing an optical channel through which data bursts can be transmitted, after an adequate delay, from source to final destination without any optical-electrical-optical (OEO) conversion [2], [3]. OBS, like other switching paradigms, does not perform well in overloaded scenarios and can present low reliability 
since it generally uses one-way reservation protocols, where data bursts are transmitted without confirmation that resources along the path will be successfully reserved to establish the required end-to-end transparent connection. Consequently, whenever the number of simultaneous reservation attempts exceed the number of available resources some will fail and, owing to the lack of sophisticated optical buffers, will result in burst loss. Burst loss degrades the global OBS performance since dropping leads to rescheduling of lost data with significant impact on any end-to-end applications running in the network layers above, reducing its overall throughput. Therefore, minimizing burst loss is a key factor for the practical implementation of OBS networks.

Considerable effort has been devoted to the study of different methods to handle contention, including, burst scheduling, optical buffering, burst segmentation, wavelength conversion, and deflection routing [4], [5]. These are mainly reactive mechanisms driven by burst contention and requiring extra hardware and/or software components at each core node, significantly increasing their cost and complexity, leading to scalability impairments. A simple and cost efficient solution is to deploy contention mechanisms at the edge nodes. This approach has been followed by using burst assembly mechanisms [6], [7], by path selection and wavelength assignment [8], [9] or by balancing the traffic load between alternate paths [10], [11].

Path selection mechanisms at the ingress nodes can alleviate contention when compared with the shortest path (SP) routing. Although successfully used in both circuit switching and packet switching networks, SP routing does not take into consideration the traffic load offered to the network, and it often causes certain links to become congested while other links remain underutilized [10]. This is highly undesirable in bufferless OBS networks, since a few highly congested links can lead to unacceptably high burst loss values for the entire network, corroborating contention avoidance strategies as an important feature in the OBS field.

The paper is organized as follows. After an introductory section where some OBS intrinsic problems are considered together with a brief state of the art, Section 2 presents the objective of the paper and its main contribution to technological innovation. Section 3 presents the simulation model and describes the OBS network simulator. Section 4 evaluates the performance of a path selection strategy and Section 5 concludes the paper with final remarks and further work for the near future.

\section{Contribution to Technological Innovation}

The aim of this paper is to present a simulation model for the evaluation of preventive routing strategies intended to minimize the global network contention and the overall burst loss through the adoption of optimized path selection strategies. Our approach uses only topological information to be integrated in an integer linear programming (ILP) formulation from which optimized routes are obtained. These routes can be used alone, as single-path static routes to provide load-balancing without the need for additional control messages with regard to link status or, alternatively, combined with dynamic contention resolution schemes (deflection or segmentation) and used occasionally as a default routing scheme to assume whenever the network needs to recover from instability, favoring the network resilience. 
This offline source routing approach presents the following a priori advantages: no extra hardware or software components are required on the core nodes and no network flooding with signaling messages resulting from (over)active link state update protocols. Moreover, with this approach there is also no place for out-of-order arrivals, a frequent disadvantage of multipath routing schemes typically requiring large memories at the edge nodes for re-ordering operations. These are distinguishing qualities to make the architecture of the OBS nodes less complex, contributing to reduce both their cost and scalability impairments.

\section{Simulation Methodology}

The simulation model described in this paper is deployed in two stages. The first stage, which comprises the determination of routing paths, is the optimization stage and was developed with the CPLEX optimizer. In this stage a routing problem is formulated using ILP, which is a widely used approach to address both high level and system level synthesis. Taking into account the computation times involved (which have been found in a range between tens of seconds and some minutes), the relatively infrequent update requests expected from changes in the OBS backbones whose topologies typically last for long time scales, and the quasi-stationary aggregate traffic demands at optical backbones, which are expected to change relatively slowly [12], this approach can be considered feasible for the real production of OBS networks by means of an operation process to be executed during its initial setup phase. The second stage, which comprises the application of the routing solution, is the simulation stage in stricto sensu, where the optimized paths produced on the first stage are incorporated into an OBS network simulation model.

\subsection{First Stage - Routing Path Determination}

Several optimization strategies for contention avoidance have been under evaluation for which different routing paths were calculated: minimizing the Max Congested Link (MCL), based on the idea that the more a certain link is included in the chosen paths for source-destination pairs, the highest the blocking probability can be [13]; minimizing the Maximum End-to-end Congested (MEC) path, based on the idea that blocking may occur at any link traversed by a burst along the path [13]; and two strategies considering the streamline effect [12], a reported phenomenon unique to OBS networks wherein bursts traveling in a common link are streamlined and do not contend with each other until they diverge. One of these strategies, entitled Streamline-Based Preplanned Routing with no Pre-determined Paths (SBPR-nPP) was recently published in [14] and, as an example, its ILP formalization will be succinctly presented next. The solution of this ILP problem will be used to drive source routing decisions on the second stage of this simulation methodology.

SBPR-nPP strategy. Let $G(N, L)$ be a network graph where $N$ is the set of nodes and $L$ is the set of links, and let us define a path over which a burst must travel, $v$, as a connected series of directed links, written as $v: s(v) \rightarrow d(v)$, from source node $s(v)$ to destination node $d(v)$. The set of paths that can be used by a burst from $s$ to $d$ is defined as $V_{s, d}=\{v: s(v) \rightarrow d(v) \mid s=s(v), d=d(v)\}$ and the set including all $V_{s, d}$ is defined 
as $V$. We also define $p_{l}^{v}=1$ if link $l \in L$ is included in $v, p_{l}^{v}=0$ otherwise. When taking a specific node as a reference point, a link coming out of that node can be denoted as $l_{\text {out }}$ and a link coming into that node can be denoted as $l_{\text {in }}$. The total number of elements in $N, L$ and $V$ is denoted by $\mathrm{N}, \mathrm{L}$ and $\mathrm{V}$. In this strategy the algorithm must find a route for burst delivery between each $s \rightarrow d$ pair of nodes, which means that no pre-determined selection of routes exists. The main goal of the next objective function is to minimize $\zeta_{M A X}$, a global bound that stores the highest number of routes competing for an output link. The second component of the objective function is a secondary goal to find routes having a small number of hops and also to avoid loops.

$$
\text { Minimize } \zeta_{M A X}+\frac{1}{\mathrm{LN}(\mathrm{N}-1)} \sum_{s, d \in N} \sum_{l \in L} \theta_{l}^{s, d}
$$

subject to

$$
\begin{gathered}
\sum_{l_{\text {out }} \in L: s\left(l_{\text {out }}\right)=i} \theta_{l_{\text {out }}}^{s, d}-\sum_{l_{\text {in }} \in L: d\left(l_{\text {in }}\right)=i} \theta_{l_{\text {in }}}^{s, d}=\left\{\begin{array}{rr}
1 & \text { if } i=s \\
-1 & \text { if } i=d \\
0 & \text { otherwise }
\end{array}\right. \\
\forall s, d \in N, \forall i \in N \\
\zeta_{i} \geq \sum_{s, d \in N} \theta_{l_{\text {out }}^{s, d}, \forall i \in N, \forall l_{\text {out }} \in L: s\left(l_{\text {out }}\right)=i}^{\zeta_{M A X} \geq \zeta_{i}, \forall i \in N} \\
\theta_{l}^{s, d} \in\{0,1\} ; \text { non - negativeinteger }: \zeta_{i}, \zeta_{\text {MAX }}
\end{gathered}
$$

where $\theta_{l}^{s, d}$ is a binary variable that indicates if the route for the s-d pair of nodes, used to carry bursts, uses link $l$. Constraint 2 guarantees flow conservation of every route. For a specific node $i$, constraint 3 stores in $\zeta_{i}$ the highest number of selected routes competing for an output link, and constraint 4 stores in $\zeta_{M A X}$ the maximum value of all $\zeta_{i}$. The main goal of the objective function is to minimize $\zeta_{M A X}$, the global bound.

\subsection{Second Stage - Routing Path Application}

The application of the routing paths calculated in the first stage is done by simulation using an OBS model developed within the OMNeT++ simulation environment [15] and some programming effort in $\mathrm{C}++$.

The functional architecture of our OBS model has the same characteristics of the one presented in [3] assuming that each node can support both new input traffic generated by the client networks as well as in transitu traffic passing all-optically from source to final destination. This study uses the following three networks: ARPAnet, NSFnet, and COST239 whose topologies are presented in Fig. 1. The nodes are connected by optical fibers with 16 wavelengths per link and a transmission capacity of 10 Gbits per second (per wavelength). The adopted traffic assumes a Poisson pattern 
with a threshold-based assembly method, generating bursts with size 100 Kbytes. Bursts are forwarded through the core backbone reproducing the relevant actions of the Just-Enough-Time (JET) [2] scheduling scheme. The control information processing time is assumed to be $10 \mu$ s per core node, although other values from $12.5 \mu$ s to 1 $\mu$ s could be adopted depending on the technology in place.

The model employs source routing, in which a complete routing decision is taken at the ingress edge node. The path over which the burst must travel is carried on the setup message that precedes the transmission of each data burst and will not be modified by downstream nodes. The adopted path is fetched from the edge node's routing tables previously populated by the results of the path selection strategy discussed in the Section 3.1. Core nodes do not employ any buffering in the data path and they do not use deflection, but full wavelength conversion is assumed. Thus, a burst is blocked only if there are no free wavelengths available to accomplish the 'next hop' on a predetermined path to a certain destination. If scheduling fails the burst is simply dropped and no further contention resolution method is adopted. Together with the network topology description, the simulation model is essentially composed of OBS capable nodes interconnected by optical fibers. The model is based on two main modules, Edge Node and Core Node, with snapshots depicted in Fig. 1. These modules will be presented next using their functional perspective.

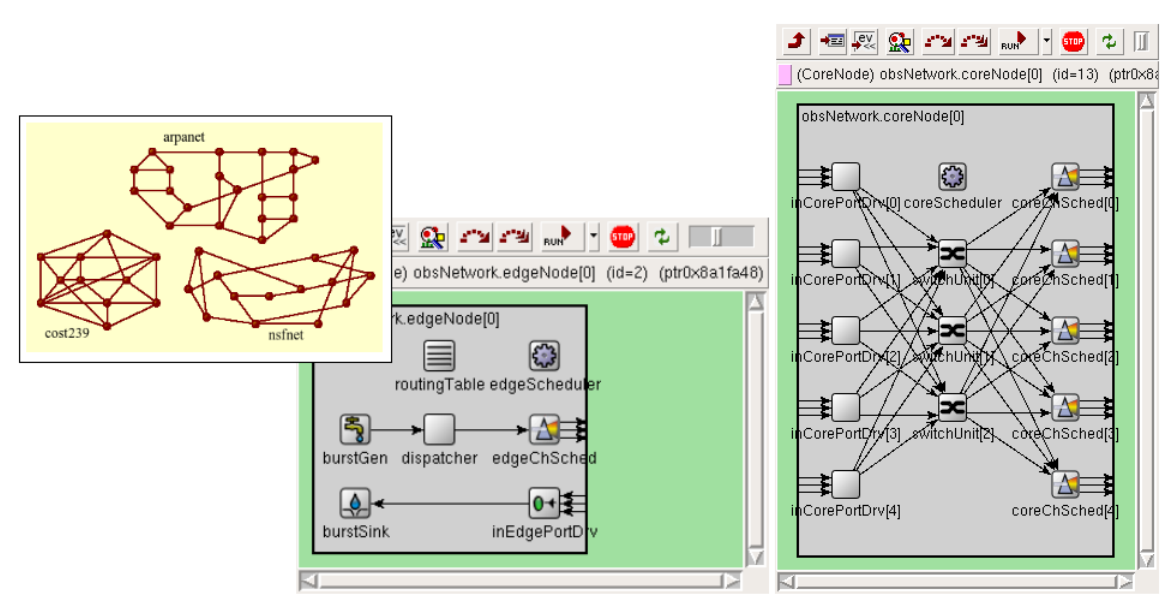

Fig. 1. Network topologies, and snapshots of edge node and core node (left to right)

Edge Nodes. Used to connect multiple subnetworks running on top of legacy link layer protocols to the OBS network. They can be considered either as ingress or egress nodes. When acting as ingress nodes, they are responsible for aggregating the incoming packets into bursts, for taking the initial (and here also permanent) routing decision, and for scheduling the bursts for transmission on outgoing channels. When acting as egress nodes, they perform the inverse operation, i.e., burst disassembly and packet forwarding to upper layers. In our model we assume the burst as the basic transport unit of interest. Hence, the issue of the packet aggregation policies is considered out of scope. Thus, whenever a Poisson process timer expires, a new burst is 
generated, a destination address is chosen at random between all other nodes in the network, a route to the destination node is taken from the source node's optimized routing table and an initial wavelength is randomly selected among the free ones. The burst is then retained on a system queue organized by destination address and the signaling process starts with the sending of a setup message on the appropriate dedicated channel. The setup message is always transmitted before the corresponding burst and apart from it by an adequate offset time. The model calculates this offset time in order to allow this message to be processed at each subsequent node before the burst arrival and in such a way that an optical path can be properly reserved for burst delivery.

Core Nodes. Responsible for processing setup messages, for switching the bursts from an input to an output port without OEO conversion and for handling contentions. Signaling in OBS is typically implemented using one out-of-band channel $\left(\lambda_{0}\right.$ is used in this model). Several signaling schemes have been proposed for burst scheduling but Just-in-Time (JIT) and Just-Enough-Time (JET) are two of the most popular protocols in OBS networks. These are both one-way and source initiated signaling schemes, which means that bursts are sent to the core network without waiting for acknowledgments regarding the success or failure of the reservation attempts. Although closely related, they differ on the duration of the reservations. JIT uses immediate reservation with the data channel being reserved immediately since the moment the setup message reaches the node, while JET delays the channel reservation until the burst arrival. This technique, together with the implicit release, makes JET more efficient than JIT regarding bandwidth utilization, resulting in lower blocking rates and low end-to-end delay [5]. The study presented here assumes a JET-kind behavior scheme. Together with burst forwarding without leaving the optical domain, core nodes are also responsible for taking contention resolution actions. Contention occurs when multiple bursts from different sources are destined for the same output port at the same time [5]. Adding to the initially path selection strategies adopted on the edge nodes, the handling of burst contentions by the core nodes assume that they are equipped with devices having full wavelength conversion capability. This means that no end-to-end wavelength continuity constraint exists, and that any incoming wavelength can be shifted to any outgoing wavelength. As a result, only if there is no wavelength available on the output port the burst will be dropped without any further contention resolution action.

\section{Performance Evaluation}

For each network topology represented in Fig. 1 simulations were done using a specifically developed OBS simulator, assuming similar conditions with regard to the total amount of bursts generated per source node $\left(10^{6}\right)$, arrival patterns and load variations. Results comparing the performance of the proposed path selection scheme with the SP routing are plotted in Fig. 2 with burst loss values normalized to the number of bursts that enter the network backbone against the average load. The plotted values of SBPR-nPP are always bellowing SP. This means that network performance can profit 

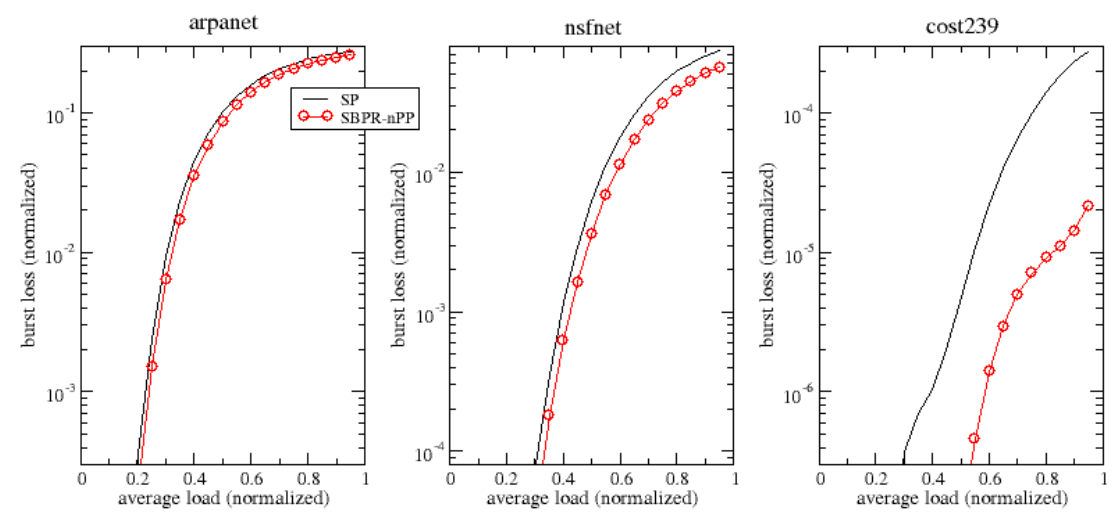

Fig. 2. Evaluation of SBPR-nPP vs. SP with normalized burst loss against average load

from balancing the potential path-load at the output links with these strategy. The graphics show also a progressively larger advantage for our algorithm following the increase of the physical connectivity parameter, with best results obtained for the COST239 European topology. This happens, because the higher connectivity of this network allows obtaining alternative paths to spread the potential traffic without extending them too much in terms of hop count. But even for the American ARPANET topology, which presents much lower connectivity values, a small benefit can be obtained with this approach.

\section{Conclusion and Further Work}

We consider the problem of routing path selection in OBS networks to minimize the overall burst loss due to resource contention of bursts aimed at the same output links. We take a preventive TE approach to provide source routing with minimized contention using only topological information and, in the case of the presented example, considering also the streamline effect. The proposed algorithm performs significantly better than the SP routing, with best performance obtained for networks characterized by having high physical connectivity.

Several research directions can be distinguished for future work. Among them, the adoption of combined routing strategies depending on traffic load variations can result in better performance. Another improvement can exploit the combined use of this static routing approach with the three basic deflection domains (time, space and wavelength) for contention resolution.

Acknowledgments. The work reported in this paper was supported in part by FCT within CEOT (unit 631) at the University of Algarve, Portugal, under the project PTDC/EEA-TEL/7168/2006. 


\section{References}

1. Qiao, C., Yoo, M.: Optical Burst Switching (OBS): A New Paradigm for an Optical Internet. Journal of High Speed Networks 8(1), 69-84 (1999)

2. Chen, Y., Qiao, C., Yu, X.: Optical Burst Switching: A New Area in Optical Networking Research. IEEE Network 18, 16-23 (2004)

3. Xiong, Y., Vandenhoute, M., Cankaya, H.: Control Architecture in Optical Burst-Switched WDM Networks. IEEE JSAC 18, 1838-1851 (2000)

4. Li, J., Qiao, C.: Recent Progress in the Scheduling Algorithms in Optical-Burst-Switched Networks. OSA Journal of Optical Networking 3, 229-241 (2004)

5. Jue, J.P., Vokkarane, V.M.: Optical Burst Switched Networks. Springer, Heidelberg (2005)

6. Vokkarane, V.M., Jue, J.P.: Prioritized Burst Segmentation and Composite BurstAssembly Techniques for QoS Support in Optical Burst-Switched Networks. IEEE JSAC 21, 1198-1209 (2003)

7. Jin, M., Yang, O.W.: Provision of Differentiated Performance in Optical Burst Switching Networks Based on Burst Assembly Processes. Computer Communications 30, 3449-3459 (2007)

8. Zhang, Q., Vokkarane, V.M., Jue, J.P., Chen, B.: Absolute QoS Differentiation in Optical Burst-Switched Networks. IEEE JSAC 22, 1781-1795 (2004)

9. Teng, J., Rouskas, G.N.: Wavelength Selection in OBS Networks Using Traffic Engineering and Priority-Based Concepts. IEEE JSAC 23, 1658-1669 (2005)

10. Teng, J., Rouskas, G.N.: Traffic Engineering Approach to Path Selection in Optical Burst Switching Networks. OSA Journal of Optical Networking 4, 759-777 (2005)

11. Li, J., Mohan, G., Chua, K.: Dynamic Load Balancing in IP-over-WDM Optical Burst Switching Networks. Computer Networks: The International Journal of Computer and Telecommunication Networking 47, 393-408 (2005)

12. Chen, Q., Mohan, G., Chua, K.: Route Optimization in Optical Burst Switched Networks Considering the Streamline Effect. Computer Networks (2008)

13. Barradas, A.L., Medeiros, M.C.R.: Edge-Node Deployed Routing Strategies for Load Balancing in Optical Burst Switched Networks. ETRI Journal 31(1), 31-41 (2009)

14. Barradas, A.L., Medeiros, M.C.R.: Pre-planned Optical Burst Switched Routing Strategies Considering the Streamline Effect. Photonic Network Communications (2009), doi:10.1007/s11107-009-021-y

15. Varga, A., Hornig, R.: An Overview of the OMNeT++ Simulation Environment. In: 1st Int. Conference on Simulation Tools and Techniques, SIMUTools 2008, Marseille (2008) 